\title{
Silicon-on-insulator mid-infrared planar concave grating based (de)multiplexer
}

\author{
M. Muneeb ${ }^{1,2,3^{*}}$, X. Chen ${ }^{4}$, E. Ryckeboer ${ }^{1,2}$, A. Malik ${ }^{1,2}$, G. Z. Mashanovich ${ }^{4}$ and G. Roelkens ${ }^{1,2}$ \\ ${ }^{1}$ Photonics Research Group, Department of Information Technology, Ghent University - IMEC, \\ Sint-Pietersnieuwstraat 41, 9000 Ghent, Belgium \\ ${ }^{2}$ Center for Nano- and Biophotonics (NB-Photonics), Ghent University, Belgium \\ ${ }^{3}$ COBRA Research Institute, Eindhoven University of Technology, P.O. Box 513, Eindhoven 5600 MB, \\ The Netherlands \\ ${ }^{4}$ Optoelectronics Research Centre, Faculty of Physical and Applied Sciences, University of Southampton, Southampton, United Kingdom \\ Email:muhammad.muneeb@intec.ugent.be
}

\begin{abstract}
The design and characterization of a silicon-on-insulator planar concave grating based (de)multiplexer operating at $3.8 \mu \mathrm{m}$ is reported. Low insertion loss $(\approx 1.6 \mathrm{~dB})$ and good crosstalk characteristics $(\approx 19 \mathrm{~dB})$ are demonstrated.
\end{abstract}

Index Terms - Silicon photonics, wavelength selective devices, mid-infrared, laser light engines

\section{INTRODUCTION}

Silicon photonic waveguide circuits were originally conceived to be used for datacommunication and telecommunication applications but a myriad of other application domains have emerged in recent years, including the use of these waveguide circuits for sensing applications [1] and biomedical instrumentation [2]. Spectroscopic sensing applications, which allow analyzing the content of gas or liquid samples of interest by probing their absorption spectrum, would benefit from the extension of the wavelength range supported by the silicon-on-insulator material platform, since the absorption cross-sections of the molecules of interest become much stronger in the mid-infrared $(2.5-8 \mu \mathrm{m})$. In this paper we present the first integrated mid-infrared planar concave grating based (de)multiplexer (also known as echelle grating) realized on silicon-on-insulator, fabricated in a CMOS pilot line, targeting the $3.8 \mu \mathrm{m}$ wavelength range. This wavelength range is very close to the edge of the transparency window of the buried $\mathrm{SiO}_{2}$ layer [3], which defines the actual transparency window of the silicon-on-insulator material platform. The developed (de)multiplexer can find applications in future integrated spectroscopic sensor systems, miniature spectroscopic telescope systems (given the atmospheric transmission window of $3-5 \mu \mathrm{m}$ ) or as a wavelength multiplexer for future quantum cascade / inter-band cascade laser light engines.

\section{DESIGN AND FABRICATION}

Figure 1 shows a schematic view of the designed PCG with important layout parameters. The design is based on the Rowland geometry with one stigmatic point [4] where different channel responses are simulated using scalar diffraction theory [5]. The central design wavelength is $3.8 \mu \mathrm{m}$ for TE polarization with channel spacing of $10 \mathrm{~nm}$. The single input and eight output apertures are positioned on a circle of radius
$866 \mu \mathrm{m}$ and DBR-based curved grating facets are placed on a circle of radius $1732 \mathrm{um}$. The spacing between output apertures is $9 \mathrm{um}$. The input aperture angle is at 34 degrees while output apertures are inclined at 37 degrees. Distributed Bragg reflectors (DBRs) have period of $880 \mathrm{~nm}$ with a fill factor of 50 $\%$.

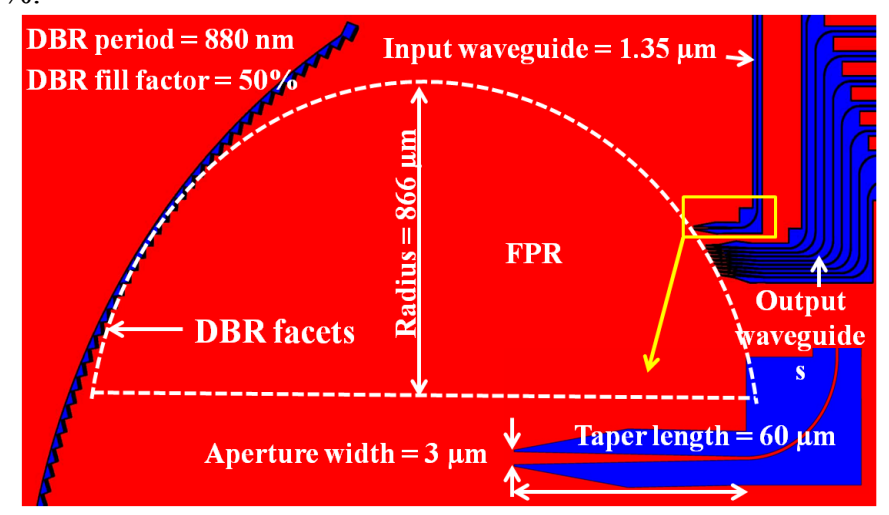

Fig. 1. Schematic view of planar concave grating (de)multiplexer.

For fabrication we used a multiple project wafer run service offered by imec, Belgium through ePIXfab [6]. A so called advanced passives process on $200 \mathrm{~mm}$ SOI wafers with $220 \mathrm{~nm}$ of crystalline silicon (c-Si) on top of $2000 \mathrm{~nm}$ of buried oxide is used. First $5 \mathrm{~nm}$ of thermal $\mathrm{SiO}_{2}$ is grown after which $160 \mathrm{~nm}$ of amorphous silicon (a-Si) is deposited using a low-pressure chemical vapor deposition process. The $5 \mathrm{~nm}$ of thermal $\mathrm{SiO}_{2}$ serves as protective layer for the underlying c-Si during the waveguide etching. On top of the a-Si $10 \mathrm{~nm}$ of $\mathrm{SiO}_{2}$ and $70 \mathrm{~nm}$ of $\mathrm{SiN}$ are deposited using plasma enhanced chemical vapor deposition process. This SiN layer serves as a hard mask for the waveguide etching. The wafer stack is then annealed at $750{ }^{\circ} \mathrm{C}$ for 30 minutes, which converts the a-Si to poly-silicon ( $\mathrm{p}-\mathrm{Si}$ ). This wafer stack is used for waveguide circuit fabrication using $193 \mathrm{~nm}$ deep UV lithography and halogen based selective dry etching. To define photonic integrated waveguide circuits in the $380 \mathrm{~nm}$ thick Si device layer (160 nm p-Si and $220 \mathrm{~nm} \mathrm{c-Si}$ ) different etch steps are available. For the planar concave grating (de)multiplexer presented in this paper we make use of $160 \mathrm{~nm}$ etch and $230 \mathrm{~nm}$ etch. Access waveguides as well as input/output apertures are fabricated using $160 \mathrm{~nm}$ etch while the 230nm etch step is used for input/output grating couplers and distributed Bragg reflectors in the planar concave grating (de)multiplexer. 


\section{CHARACTERIZATION}

The waveguide circuit is characterized using grating-based fiber-chip interfaces connected to input and output ports of PCG. The details of the measurement setup are discussed in [7], to which two changes were made. Firstly, the source now consists of a tunable quantum cascade laser (tuning range: 3725 $\mathrm{nm}-3895 \mathrm{~nm}$ ) from Daylight Solutions and secondly instead of butt coupling to the waveguide structures vertical fiber-chip interfacing is used. Figure 2 shows the transmission measurement results. The transmission spectra are normalized to a reference waveguide so that only the insertion loss of PCG channels themselves is shown.

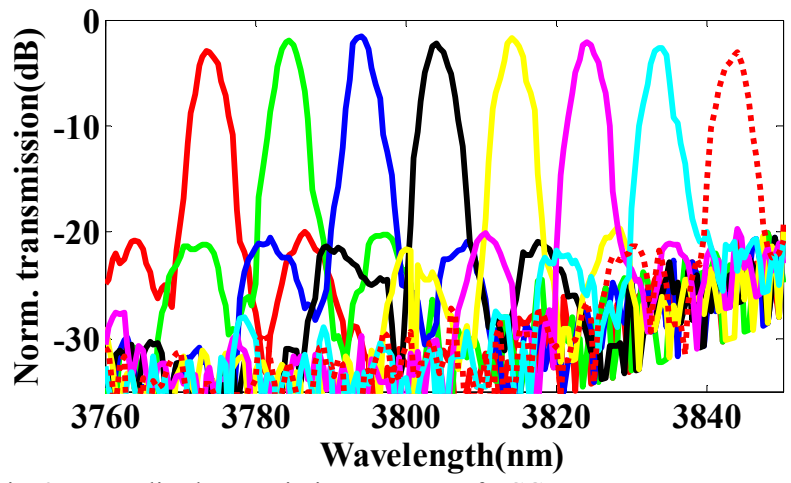

Fig. 2. Normalized transmission response of PCG.

To show the validity of the simulation method based on scalar diffraction theory, simulated and measured spectra of the first channel are compared in figure 3 . Both spectra are normalized to $0 \mathrm{~dB}$. Spectral shapes around the central wavelength overlap almost perfectly. Comparison of the side lobes is not possible as the level of these side lobes are mainly due to fabrication imperfections, which the simulation method used does not take into account.

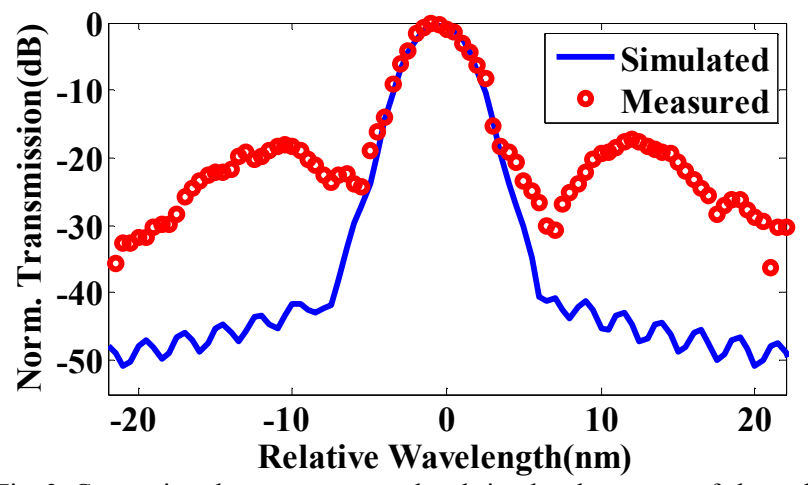

Fig. 3. Comparison between measured and simulated response of channel 1.

Loss for central channels is less than $1.75 \mathrm{~dB}$ of which major contribution comes from the fabrication imperfection of the DBRs. SEM inspection shows the fabricated fill factor to be 38 $\%$ instead of designed $50 \%$ which gives almost $0.8 \mathrm{~dB}$ reflection loss. Other loss contributions come from corner rounding and non-verticality (10 degrees in this case) of grating facets. Crosstalk is better than $19 \mathrm{~dB}$ for all channels which is again limited by fabrication imperfections especially the thickness non-uniformity of the free propagation region. To emphasize the similarities and differences between different channels figure 4 plots six output channels together by shifting them by integer multiples of the channel spacing. Spectral shapes of channels as well as side lobe structures overlap very well. The side lobes are nearly independent of channel number within the same PCG.

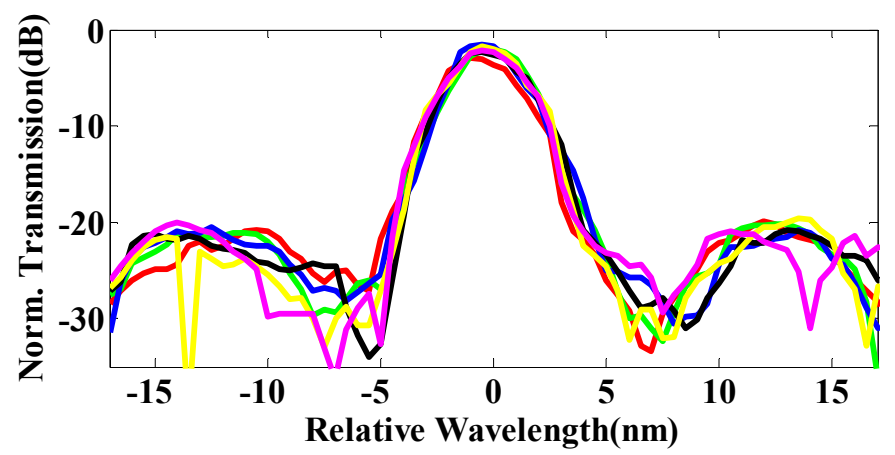

Fig. 4. Overlapped spectra of six channels.

\section{CONCLUSION}

In this paper we demonstrated the first planar concave grating based (de)multiplexer, implemented on a silicon waveguide platform at center wavelength of $3.8 \mu \mathrm{m}$. The device was fabricated in a CMOS pilot line, illustrating the potential for large-volume and low-cost manufacturing of such circuits. Moreover, since the imec advanced passive process is offered as a multi-project wafer run service, these mid-infrared circuits can be designed and fabricated alongside near-infrared photonic integrated circuits.

\section{ACKNOWLEDGMENT}

This work was carried out in the framework of the FP7-ERC-MIRACLE project. Goran Z. Mashanovich would like to acknowledge support by the Royal Society through his Royal Society Research Fellowship.

\section{REFERENCES}

[1] E. Hallynck and P. Bienstman, "Integrated optical pressure sensors in silicon-on-insulator," IEEE Photonics Journal 4(2), 443-450 (2012).

[2] M. C. Estevez, M. Alvarez, and L. M. Lechuga, "Integrated optical devices for lab-on-a-chip biosensing applications," Laser \& Photonics Reviews 6(4), 463-487 (2012).

[3] Richard A. Soref, Stephen J. Emelett, and Walter R. Buchwald, "Silicon waveguided components for the long-wave infrared region," J. Opt. A: Pure Appl. Opt. 8(10), 840-848 (2006).

[4] R. Marz. Integrated Optics, Design and Modeling. (Artech House Inc., 1994)

[5] K. A. McGreer, "Diffraction from concave gratings in planar waveguides," IEEE photonics Technology Letters, 7(3), 324-326 (1995).

[6] http://www.epixfab.eu/

[7] Goran Z. Mashanovich, Milan M. Milošević, Milos Nedeljkovic, Nathan Owens, Boqian Xiong, Ee Jin Teo, and Youfang Hu, "Low loss silicon waveguides for the mid-infrared," Optics Express 19(8), 7112-7119 (2011). 